International Journal of Aging and Human Development, 41(2), 69-107, 1995.

\title{
CHANGING THE WAY WE TALK WITH ELDERS : PROMOTING HEALTH USING THE COMMUNICATION ENHANCEMENT MODEL
}

\author{
Ellen Bouchard Ryan, Sheree D. Meredith, \\ Michael J. MacLean, and J. B. Orange
}

\begin{abstract}
Good communication is an essential component of optimal delivery of health care and health promotion efforts. In this article, we address the communication predicament fac ed by older adults when their opportunities for optimal care are limited by inappropriate communication with formal care providers. We then introduce the Communication Enhancement Model which promotes health in old age by stressing recognition of individualized cues, modification of communication to suit individual needs and situations, appro priate assess - ment of the health/social problems, and empowerment of both eiders and providers. Applications of the Communication Enhancement Model are discussed for two high-risk groups (elders from ethnocultural communities and elders with dementia) to show how it can function as a guide for the development and evaluation of educational interventions with health and social professionals working with elders.
\end{abstract}

Good communication is important in achieving health across one's lifespan. Moreover, it is central in the delivery of health care and in health promotion efforts of formal care providers. In this article, we initially address the communication predicament which limits the opportunities of older adults to optimize their health. We then present a communication enhancement model to promote health in old age, and we elaborate on the role of formal care providers from various professional disciplines (referred to here as providers) in participating in this health-promoting communication process. Finally, we use two case studies to highlight the application of this model for elders who are especially vulnerable to the communication predicament we describe.

\section{COMMUNICATION PREDICAMENT OF ELDERLY PEOPLE}

In late life, older adults frequently experience chang es which threaten their communication skills [1-3]. Hearing and vision impairments, slower processing of information, and memory difficulties affect many otherwise healthy elders, while neurological, depressive, and physical illnesses as well as medications, can also influence communication. Moreover, reduced contacts with relatives and friends may limit the opport unity to exercise conversational skills for a number of older adults.

At the time in their lives when older adults need an especially supportive and stimulating interpersonal environment, many individuals experience a communication pre dicament. This predicament arises when older adults, experiencing changes in their individual skills, have to overcome extra barriers imposed by their conversational partners [4, 
5]. Figure 1, adapted from Ryan et al. [5], depicts the manner in which the communication predicament of older adults can be influenced by the interpersonal environment in which they attempt to communicate.

The communication predicament depicted in Figure 1 begins when the provider encounters the older person. First encounters may begin with the name and chronological age on a list of client appo intmentsmso that inferences regarding the impact of old age can be activated well before the initial meeting. Whether actual age is known or not, the provider can guess the person's age on the basis of cues such as appearance (e.g., white hair), behaviors (e.g., hearing difficulty), and roles (e.g., retiree).

Following the cycle in Figure 1, one sees that recognition of old age then elicits stereotyped expectations of older people. Low er expectations of older adults still persist in terms of intellectual and productive competence as well as health status [6-8]. Older adult speakers have been shown to elicit lowered evaluations of competence as compared to younger adult speakers [6,9]. Moreover, age-biased interpretations of the quality of their communication (i.e., reactions to changes in spe ech rate or effectiveness of message) have been observed $[9,10]$.

The next step in the cycle is inappropriate communication accommodation toward the older person through the modification of speech and other communication behaviors based on the stereotypes elicited. Within everyday communication, age biases are revealed in the way interlocutors talk with elders $[5,11]$. Inappropriate modifications are elicited, not only by actual age-associated changes in communicative competence, but also by stereotyped expectations of limitations based on age alone or in combination with visible handicap (e.g., cane or wheelchair) or dependent role (e.g., nursing home resident or hospital patient).

Speech modifications include slow er speaking rate, exaggerat ed intonation, high pitch, increased loudness, greater repetition, simpler vocabulary, and reduced grammatical complexity [ 12]. Further, the literat ure also identifies the occurrence of baby talk, patronizing talk, controlling parental talk, feigned deference, avoidance of talk due to anticipated verbosity or generation gap, less listening to concerns, restricted range of topics, age-biased interpretation of elder's comments, and discussion of the older person's problems with a third party as if the elder were not present $[11,13,14]$. If behavioral modifications are based on generalizations rather than individual skills, these alterations in style of communication often convey a fundamental lack of respect for the elderly person $[12,13]$. Despite the good intentions usually asso ciated with such speech, these modifications can seriously reduce the opportunities for the older person to participate in conversation, to provide or obtain needed information, and to gain satisfaction from an encounter.

Moreover, there is a tendency for such modifications to reinforce agestereotyped behaviors and to exaggerate behavioral deficits of frail elders. Baltes and Wahl review their field research indicating that independent behaviors of institutionalized and community residing elders in daily self-care activities are ignored by caregiving staff while dependent behaviors receive positive reinforcement [15]. This implicit reinforcement of dependence in care for elders in institutions and in the community can even occur in contradiction to the particular overt purpose of care [16.].

Following through the loop in Figure 1, older people can experience negative changes from conversational encounters, which further reduce their chances for successful communication and good care in the future. That is, elders unsure of their current level of 
abilities may observe how they are treated and infer from this social mirror that they are in decline. Such inferences can directly reduce self-esteem and effort and also indirectly influence future performance by leading to decisions to withdraw from activities that have been enriching and challenging of their abilities [17]. Thus, future encounters with this elder may begin with a first impression of a person who seems even "older" or more impaired in terms of behaviors and roles.

\section{COMMUNICATION ENHANCEMENT MODEL FOR INTERACTIONS WITH ELDERLY PEOPLE}

The negative impact of the communication predicament (Figure 1) on an older individual's health and well-being has been well-documented. If, as Kreps stat es, "The diagnosis, education, and treatment of health problems is accomplished through the maintenance of communication relationships between the providers and consumers of health care" [ 18, p. 210], then there is an urgent need for formal care providers and educators to confront this predicament. In this section, we present the Communication Enhancement Model which uses a health promotion framework and interventions as appropriate vehicles to accomplish the objective outlined by Kreps [18].

\section{Health Promotion Framework and Interventions}

The World Health Organizat ion defines health promotion as "... the process of enabling people to increase control over, and to improve their own health" [19, p. 101]. This definition includes the individual and the social environment, and is consistent with Green and Raeburn who argue that "any realistic view of the determinants of health and of appropriate action for health promotion requires a balance of individual, community, institutional, so cietal and political per specfives" [20, p. 154]. A dual focus on the individual and the environment is compatible with an ecological perspective of health and well-being which Compton and Galaway describe as a need for balance between an individual's capacity, opport unities, and motivation on the one hand and the expectations, resources, and opportunities provided by the environment on the other [21]. The objective of intervention by health care professionals lies in establishing and maintaining this balance.

The implications for intervention are three-fold: 1) change must be directed toward both the individual and the environment to be most effective; 2) Health Promotion strategies must encompass diverse social, health, and economic programs; and 3) current resource allocation patterns must be restructured to support this broader emphasis. Presently, the major focus of the health care delivery system is on intervention at the patient/professional level. This is reflected in the dyadic nature of the communication predicament described earlier.

A Health Promotion framework simultaneously increases the complexity of our understanding and approach to health care. Interventions must occur not only within the dyadic relationship, but also outside of it [22-24]. Epp, in his policy document entitled, "Achieving Health For All: A Framework for Health Promotion" [25], proposes three intervention domains: self care, mutual aid, and healthy environments.

\section{Self Care}

Self care interventions, defined as "decisions and actions individuals take in the interest of their own health" [25, p. 7], focuses on empowering individuals by ensuring that they have 
adequate information, skills, opport unities, and motivation to decide and act. Older individuals, for example, may benefit when information is presented in non-traditional forms which surmount barriers created by language, literacy, visual, or auditory impairments. Because of their so cialization to accept the authority of the health care system and professionals [26], the current cohort of elders may also require substantial support in the development of the skills and motivation to ask questions, explore options, and make health care decisions. A variety of successful self-care intervention programs have been documented, including: self-advocacy training for older adults [27], assertiveness training to enhance selfconcept and promote maximum use of resources [28], and develo pment of the skills of family car egivers in negotiating the health care system [29].

\section{Mutual Aid}

Mutual aid interventions are defined as "people's efforts to deal with their health concerns by working to gether.., helping each other, supporting each other emotionally, and sharing ideas, information and experiences" [25, p. 7]. While spousal support might be considered the most fundamental and least formalized form of mutual aid, a number of informal networks, support gro ups, and voluntary organizations have developed to fill this role. Many of these groups sup port their members through the provision of education, advocacy, and social support. As such, they are instrumental in enhancing people's capacity and mo tivation to interact effectively with formal care providers [25]. Similarly, mutual aid mechanisms can be helpful to formal care providers. Membership on interdisciplinary teams, participation in continuing education programs, and access to ethics committees, or resources such as geriatric consultation teams can be viewed as potential sources of mutual aid for providers.

\section{Healthy Environment}

The third health promotion int ervention, the creation of healt hy environments, is defined as "altering or adapting our social, economic, or physical surroundings in ways that will help not only to preserve but also to enhance our health" [25]. In developing a communication model that promotes health, one needs to focus on eliminating barriers created by the health care environment. Interventions must be directed toward ensuring appropriate, individualized responses to older individuals by caregivers [26, 30]. Providers must unders tand and support a process of empowering the older client. Equally important is the adaptation of the psychosocial and physical environments in which the exchange occurs in order to provide maximum opportunity for communication [31 ].

Within the health promotion framework of self care, mutual aid, and healthy

environments, one must also examine system barriers which may have a significant negative impact on the communication between elders and providers.

Legislation, intake procedures, schedules, funding formulae, and assessment tools each contribute to the context in which the communication between the elder and provider occurs. The expectations, resources, and opportunities in the broader community also impact on the provider's understanding and response to the elder.

THE COMMUNICATION ENHANCEMENT MODEL EMERGING FROM THE HEALTH PROMOTION FRAMEWORK 
Figure 2 depicts the Communication Enhancement Model we have developed utilizing a health promotion framework. The cycle begins with the encounter between the older person and the provider. The provider now comes to the encounter with a different cognitive map as a result of educational interventions.

These interventions will have been directed toward increased understanding of normal aging processes and preparation to undertake new roles and utilize new skills in interacting with elders. One emphasis, for example, is on the helper's expanded role as co-advocate and partner in decision making in contrast to the traditional hierarchal relationship and practice style prevalent in most medical expert-older patient relationships [26]. In these new roles the provider must be active in understanding the broad range of variables influencing the health of an elderly person as well as in eliciting his or her expectations and wishes. As Bieseeker notes, while individuals aged sixty and over seek more information from providers, they are less likely than younger cohorts to believe in a patient's right to make decisions, ask questions, or challenge a provider's authority [32] Therefore, the provide's role must also expand to include the education and support of elders as many of them learn the new roles and skills for becoming more active participants and consumers in the health and social care systems. As a consequence, providers must develop the'ability to share knowledge in ways which enable clients and others to comprehend and effectively utilize it. An expectation that the older person may be motivated and capable of actively participating must be fostered in conjunction with the development of a greater appreciation of the diversity and potential of elders. Likewise, the older person will likely come to the dyadic encounter with altered expectations and confidence in his or her role as active participant and the skills needed to fulfill the role. This situation can be enhanced through a variety of self care and mutual aid strategies, many of which will occur outside the encounter with the provider.

Combined, these strategies and resources provide a starting point for a more individualized, responsive communication encounter between the elder and the provider. This includes app ropriate accommodation of communication matched to the older individual's needs; a physical environment that maximally supports this exchange including resources such as technical aids, written materials, audiovisual aids, and translators; and a social environment where the helper conveys genuine respect, interest, and sensitivity toward the concerns of the elder. As depicted in Figure 2, one can see the feedback nature of this positive cycle. As the provider utilizes effective communication strategies, for example, he/she develops a clearer assessment of the elder's capacity, expectations, and resources which leads to a further refinement of strat egies and enhancement of communication.

Similarly, the respectful appro ach of the provider will serve to reinforce the elder's willingness and ability to participate actively. In Figure 2, we see how this leads to a more appropriate assessment of the health concern. By utilizing an ecological health promotion framework for interpreting the information gathered, the provider and elder, together, can develop a multi-focused plan of intervention which draws resources from a variety of sectors. For example, if loneliness and social isolation are key concerns, the plan might include reassessment of heating, new communication strategies for the elder, reinvolvement in a church service group, negotiation with the family about visiting and transportation needs, and consideration of home-sharing or a move into town.

As a result of joint problem-solving, both the elder and the provider are empowered. When the elder has been actively involved in developing a plan which is well-matched to 
his/her unique situation, there is an increas ed likelihood of follow-through and success. The health, well-being, and competence of the older person are also enhanced. Similarly, the mutual nat ure of the communication can ensure that there is a balance with the provider's resources. An ecologically based plan takes into account environmental and care system constraints and also seeks opportunities for modifying these external influences to the benefit of an individual elder and similar others. Enhancements in communication, ass essment, and intervention naturally lead to increased satisfaction experienced by the provider. Most important, perhaps, is the recognition that this can prevent the suffering which is unintentionally caused when providers intervene in ways which ignore the strengths of the person [33].

Both participants can emerge from this experience better equipped to deal with the next encounter. In addition, the environment may also become more responsive to the needs of older people as a result of the provider adopting an ecological perspective and expanded role throughout this cycle. A successful example using multiple interventions is the Wise Use of Medications project of a Toronto community health center [34]. In this project, a needs assessment with seniors identified barriers to appro priate communication about medications. Subsequently, information about medication misuse was provided to seniors, and groups of seniors developed approaches for dealing with the various professionals involved. Finally, participants in various parts of the health care system worked with seniors to de velop a safe medication card and promote its use by seniors in each health care encounter.

The Communication Enhancement Model has relevance for providers from all disciplines. It is perhaps most important for those working with older clients who have special needs. This includes such groups as ethnic minorities, women, cognitively impaired individuals, and those who are physically dependent but mentally alert. We focus on two of these groups to examine the applicability of the Communication Enhancement Model.

\section{APPLICATIONS OF THE COMMUNICATION ENHANCEMENT MODEL}

\section{Promoting Health through Communication withElderly People from Ethnocultural Communities}

The communication difficulties that care providers experience with elderly people from non-majority ethnocultural communities go beyond those existing between providers of social and health care services and elderly people of the majority culture. These specific communication difficulties relate to issues of language and culture of the elderly person vis-avis formal care, in combination with physiological, psychological, and sociocultural cues that providers experience in their work with elderly people in general. These difficulties arise because the vast majority of the providers who give care to elderly people from non-majority ethnocultural communities are from a different cultural background than these elderly people, and they are working in a system of care which tends to promote a unidimensional cultural approach to the provision of services [35]. It is important to develop a more positive model of communication in care for elderly people from ethnocultural communities because formal care providers in co untries such as Canada, the United States, England, and Australia will increasingly come in contact with these elders. The numbers of elderly people from ethnocultural communities especially those aged eighty and over are increasing at greater rates than other elderly people in these countries [35-37]. Furthermore, elders who speak English as 
a second language may begin to lose this ability and become more reliant on their mother tongue due to changes in social environments or to cognitive impairment $[38,39]$. Therefore, there is a need for a communication enhancement model with elderly people from ethnocultural communities if providers and educators are going to contribute to Epp's "Health for All" philosophy that most formal care providers would espouse.

The essential components of the Communication Enhancement Model to promote health have considerable implications for elderly people from ethnocultural communities. The issues of recognition of cues on an individualized basis, modified communication to accommodate individualized need, appropriate assessment of health and social issues, empowerment of the elderly person and the provider, and maximizing opportunities for communication clearly fit into recent strategies developed for improving communication with ethnic and minority aged people. These strategies include guarding against ethnocentric behavior, developing sensitivity to cultural issues vis-a-vis health, avoiding ethnic myths and stereotypes, practicing empathy, and being aware of individual differences within groups [39]. These strategies and the components of the Communication Enhancement Model speak to establishing a respectful relationship toward elderly people from ethnocultural communities in the provision of care.

The link between the Communication Enhancement Model and the respectful relationship with ethnic elders can be illustrated through a case study of an elderly woman from Lithuania who was in a long-term care institution of the majority culture, and who needed the skills of health care pro viders at a particularly difficult and sensitive time in the caring process--that is, when she was dying [40]. This part of the caring process is difficult for an elderly person from an ethno cultur al community because the pers on is in a linguistically and culturally foreign environment. The stress of caring for someone who is dying is exacerbated when providers are unaware of the cultural norms of the dying person. As this is a time when sensitive care is critical, this case provides a good illustration of the Communication Enhancement Model. The case will be presented from the perspective of the social worker as she had the most significant interaction with Mrs. A. during this critical period.

Mrs. A. was an eighty-seven-year-old Lithuanian widow admitted to an institution about one month before she died. She was ill with cancer, and there was very little medically that could be done for her. She had a sense that she was dying but she did not want to dwell on it so the staff of the institution simply tried to make her as comfortable as possible. She spent her adult life in the Lithuanian community in Canada so her English was poor, and she only used it to convey basic needs to the social worker. She had a son who visited daily and spoke to her in her native language. Two we eks before her death, Mrs. A. was transferred to the chronic care ward of the institution due to det eriorating health. She became unusually upset at this time; this was confusing as her behavior seemed to be unrelated to an increase in pain. The social worker suspected that there may have been psychological or sociocultural issues that were upsetting her, but she could not determine this directly as, by this time, Mrs. A. had lost the ability to converse in English. This recognition of cues for Mrs. A.'s situation led to a reassessment of the health problem which led to asking her son if there were individual or cultural issues that were contributing to her being disproportionately upset. After checking with Mrs. A., her son told the social work er that she was disturbed by the curtains around her bed because she did not want to die in the dark; she was also worried about her pain causing her to lose 
control in front of three other women who were sharing her room; she was also anxious that her son visit more frequently because she could no longer communicate in English. This information from her son allowed the social worker to make arrangements to move Mrs. A. to a private room which had considerable natural light. A nurse who spoke Lithuanian was located in the institution, and she visited Mrs. A. on a regular basis when her son was not able to be there. This permitted Mrs. A. to become calm, and to develop an increased sense of well-being. Mrs. A. died four days after she had been moved to the new room.

An analysis of this case study shows how the components of the Communication Enhancement Model to promote health can be used by a care provider to considerable effect with an elderly person from an ethnocultural community. The social worker recognized that Mrs. A.'s behavior was different from that which would have been expected from previous experience with her. This change was confusing, and, therefore, stressful to the social worker because she did not know how to use her skills to help Mrs. A. Given that the elderly woman could no longer speak English, the social worker had to modify her communication pattern to accommodate Mrs. A.'s individual needs. This modification was operationalized by developing a reciprocal relationship with Mrs. A.'s son, who could provide information leading to an appropriate assessment of the social issue that was causing such concern to Mrs. A. Note that the triad with the third person intermediary poses its own challenges to maintain respectful communication with the older client [41].

This intervention of seeking Mrs. A.'s understanding of the problem led to empowerment of the social worker (who now knew what the problem was and how to make changes in Mrs. A.'s circumstances) and to the client (who, through her son, was able to communicate her so cial and health needs). The changes evolving from the empowerment of the worker and the client led to the increased effectiveness of the worker and to optimized health and well-being of the elder at a very stressful period of her terminal stage of life. This process led to enhanced communication skills and opportunities for both the social worker and Mrs. A. because it showed that communication is much broader than simply an exchange of linguis tic information. This broader level of communication is often impossible for many health care practitioners working with elderly people from ethnocultural communities.

The key results of this process, illustrative of the Communication Enhanc ement Model, are that Mrs. A. was given some control over how she was going to die, even though it was in an institution not of her culture, and that the social worker was able to use her skills for the optimum benefit of her client, even though they were from different cultural backgrounds. The ecological perspective is highlighted here by the role of the physical/social enviro nment in the problem (shared room) and the solution. From this specific example of changing the way we talk to elders, it is clear that the Communication Enhancement Model can be used to promote health with elderly people from ethnocultural communities.

The three health promotion interventions offer some strategies to enhance communication between members of ethnocultural communities and formal care providers. In terms of self care, elders can receive education in their native language concerning how to access the formal care system and ways to get their messages across to providers. Mutual aid offers many avenues including the recruitment and training of bilingual elders to serve as cultural and health promotion interpreters for their peers and to assist the work of the small number of formal providers from the same ethnocultural communities. From a healthy 
environment perspective, home care services can be made more flexible so that they facilitate the care offered by families and other members of the care receiver's ethnocultural community, and better links can be established between the ethnocultural community and their elderly members receiving care in institutions.

\section{Promoting Health of Demented Elders and their Caregivers via Communication}

The components of the communication predicament previously outlined have far reaching impact on care providers of individuals with dementia that go beyond mere exchange of information. Communication which focuses on linguistic disturbances, negative stereotypes, and lack of respect for personhood is likely to yield a predicament of confrontation and serious negative biopsychosocial consequences such as poor health status, depression, and social isolation. This is not unlike the situation faced by elderly people from ethnocultural communities. The importance, therefore, of the Communication Enhancement Model for individuals with dementia is that it offers providers and the social and health care system strategies to o vercome biases and barriers and establishes a framework for productive exchange of personally meaningful information.

These consequences have particular significance in light of the increasing proportion of demented individuals in the ever-increasing aging segment of our population [42].

Since demented individuals suffer progressive degenerative disturbances in memory, learning, and communication, care providers working with them must be sensitive to everchanging needs and performance $[1,43,44]$. The recognition of unique profiles of performance for the demented population is imperative as their communication, memory, and social skills, even within clinical stages, are particularly diverse [45]. This heterogeneity has important ramifications for the selection of assessment protocols, int erp retation of diagnos tic information, and the development of diagnosis, prognosis, and care management plans. Optimization of health, social, and psychological status of cognitively impaired elders, using the three health promotion interventions to serve the aims of the Communication Enhancement Model, can have substantial impact in forestalling institutionalization [46].

In terms of self-care strategies, interventions focused on modifying the demented individual's linguistic communication and pragmatic features have met only with limited success $[47,48]$. Self-care strategies linked with environmental support are more promising, such as training and support for family caregivers and the use of advo cates for ongoing healthcare choices [49]. Advocates are particularly important for individuals with dementia where cognitive impairment robs them of their ability to make informed health-care choices. Advocates need to be able to communicate and effect their partners' wishes and, similarly, the health care system needs to be free to respond to these pre-established wishes. Implementation of the health care choices of demented individuals, pre-selected at a time when they were cognitively competent and in consultation with their advocate and personal physician, increases the likelihood of humane, respectful, and appropriate care.

Modification of communication within the dyadic interaction is crucial for care providers. Clinicians must be made aware not only of the variability of performance of the demented individual, but also, more importantly, of the necessity that providers make adjustments in their linguistic structures, vocal cues, nonverbal cues, and conversational style. Additional healthy environment strategies might include the use of assistive listening and vision devices and modifications which minimize background auditory, visual, and tactile noises. 
Importantly, the cognitive and social disturbances associated with dementia require providers to confront and extinguish the use of inappropriate accommodations of communication (e.g., baby talk) which support and exacerbate age-stereotyped behaviors of dependence and frailty, as previously discussed. While a considerable amount of communication adjustment is necessary to accommodate to the impaired functional capacities of demented individuals, selected reinforcement of residual communicative skills can optimize social interactions and facilitate independence [47].

With respect to mutual aid strategies, family care providers of demented individuals often need community support for information, training, and respite care. Many local communities offer individual consultation and information sessions, which include a focus on communication and management strategies for dysfunctional behavior. In addition, peer support gro ups may break down the barriers isolating family care givers fro $m$ colle agu es in similar circumstances and give them opportunities to assist each other in problem solving and in coping with the demands of such family care.

Emanating from the se examples of interventions are consequences that impact on the dyad of provider and dement ed individual, and on the health system as a whole. Positive changes emerge in the resourcefulness of care providers, their access and utilization of services as well as the manner in which the health care system promotes communicative interactions that reinforce the auto nomy and dignity of demented elders.

The follow ing case study illustrat es a combination of intervention options from the Communication Enhancement Model which were selected by care providers for an individual diagnosed with probable dementia of the Abheimer's type in the middle/moderate clinical stage:

Mr. S. was a seventy-two-year-old mechanical engineer who was dwelling in the community with his wife. Mrs. S. wished to visit a son and his family who lived out west. One of Mrs. S.'s grandchildren, with whom she was particularly close, was graduating from high school with honours and Mrs. S. desperately wanted to attend the award ceremonies. Traveling with her husband was out of the question as Mr. S. suffered terrible confusion, limited communicative effectiveness, and periodic catastrophic reactions in unfamiliar environments. Using healthy environment strategies to assist with preparation for the trip, Mrs. S. enrolled her husband in an adult day care program situated in a local home for the aged. Attendance for the first three weeks was one day a week. Mrs. S. accompanied her husband to and from the program, staying for approximately one-half hour at the beginning of each session and arriving early near the end of the day. The frequency and length of visits to the center was gradually increased while Mrs. S.'s overlap time decreased. The intention was to familiarize Mr. S. with the surroundings as the home was equipped with three respite care beds, one of which was to be his during his wife's two week trip in the coming months.

In conjunction with the enrollment, Mrs. S. discussed with the staff the findings of recent medical, geriatric, language, and communication assessments. Individualized intervention strategies were drawn up by the consulting professionals, discussed with Mrs. S. at great length. Mrs. S. then discussed them with the staff of the residence to familiarize them with Mr. S.'s skills, needs, and interests.

Consultants made themselves available to Mrs. S. and staff for further discussion when 
need ed. Healthy enviro nment and self-care strategies were reviewed, including speak ing in direct, nonliteral, declarative statements with modifiers placed after their antecedents, eliminating the use of secondary baby talk, increased use of appropriate nonverbal behavior in conjunction with verbal output, as well as limiting the number of partners and new topics in conversations.

Detailed background information regarding Mr. S.'s job history, personal interes ts in Canadian history and horticulture was also relayed to staff so that they could maximize the familiarity of personal contacts and "chat at length on mutually shared topics. The successful implementation of these and other related interventions enabled Mrs. S. to achieve maximum benefit from the trip and showed staff how selected strategies from the Communication Enhancement Model can promote optimum health of and care for individuals with dementia.

\section{Implications}

These case studies illustrate how the Communication Enhancement Model can be used to guide improved communication with vulnerable elders and to empower the $m$ and their families to work more effectively with formal care providers. Emphasizing the health promotion aims of the model can make it easier to educate staff in the importance of individualized and culturally sensitive communication.

\section{SUMMARY}

The Communication Enhancement Model to promote health with elderly people introduced in this article has considerable implications for interdisciplinary education and practice for health and social service providers. The model emphasizes care providers' multiple roles in promoting health through fost ering communication skills with their aging clients. This model indirectly addresses the unintentional suffering which may arise from inappropriate communication strategies among formal care providers to ward the older adults they serve. The model also provides a series of steps for health and social service practitioners to implement when developing communication strategies with special needs clients where interventions are particularly challenging. With these implications in mind, this model of communication to promote health can be used to contribute to positive interactions among formal care providers and their elderly clients.

Importantly, the Communication Enhancement Model incorporates the ecological perspective being sensitive to the balance between the system of care and provider-elder communication. Such a balanced appro ach promotes mental, social, and physical well-being among elderly individuals, especially vulnerable individuals such as members of ethnocultural communities and persons with cognitive impairment.

Future work is needed to evaluate the impact of communication interventions by providers on communication satisfaction and health outcomes in the elders they serve. Mor eover, educational programs within professional training or continuing educ ation should be developed and evaluated in terms of impact upon communication skills of providers and the related consequences for their elderly clients. Relatedly, the communication and health impact of specific environmental or system changes (e.g., home asse ssments versus asses sments in institutional settings; providers meeting with small groups of elders with a particular health problem versus one-to-one interactions) should be evaluated either singly or in combination 
with educational interventions aimed at providers and/or elders themselves. In terms of policy implications, the Communication Enhancement Mod el highlights the changes need ed in

provider-elder interactions within a health promotion framework and raises issues concerning professional education and service delivery.

\section{ACKNOWLEDGMENTS}

We would like to thank Phyllis Blumberg and How ard Giles for comments on an earlier draft of this article.

\section{REFERENCES}

1. K. A. Bayles and A. Kaszniak, Communication and Cognition: Normal Aging and Dementia, Little, Brown, Boston, 1987.

2. J. F. Nussbaum, T. Thompson, and J. D. Robinson, Communication and Aging, Harper, New York, 1988.

3. E. B. Ryan, Language Issues in Normal Aging, in Dementia and Communication, R. Lubinski (ed.), Mosby, Philadelphia, 1991.

4. N. Coupland, J. Coupland, H. Giles, and K. Henwood, Accommodating the Elder ly: Invoking and Extending a Theory, Language in Society, 17, pp. 1-41, 1988.

5. E. B. Ryan, H. Giles, G. Bartolucci, and K. Henwood, Psycholinguistic and Social Psychological Components of Communication by and with the Elderly, Language and Communication, 6, pp. 1-24, 1986.

6. H. Giles, N. Coupland, J. Coupland, A. Williams, and J. Nussbaum, Intergenerational Talk and Communication with Older People, International Journal on Aging and Human Development, 34, pp. 271-297, 1992.

7. M. E. Kite and B. T. Johnson, Attitudes toward Older and Younger Adults: A Metaanalysis, Psychology andAging, 3, pp. 233-244, 1988.

8. E. B. Ryan, S. Kwong See, W. B. Meneer, and D. Trovato, Age-based Perceptions of Language Performance among Younger and Older Adults, Communication Research, 19, pp. 311-331, 1992.

9. M. A. Stewart and E. B. Ryan, Attitudes toward Older and Younger Adult Speakers: Effects of Varying Speech Rates, Journal of Language and Social Psychology, 1, pp. 91-109, 1982.

10. E. B. Ryan and S. Laurie, Evaluations of Older and Younger Adult Speakers: The Influence of Communication Effectiveness and Noise, Psychology and Aging, 5, pp. 514-519, 1990.

11. N. Coupland, J. Coupland, and H. Giles, Language, Society and the Elderly, Basil Blackwell, Oxford, 1991.

12. L. R. Caporael, The Paralanguage of Caregiving: Baby Talk to the Institutionalized Aged, Journal of Personality and Social Psychology, 40, pp. 876-884, 1981.

13. E. B. Ryan, R. Y. Bourhis, and U. Knops, Evaluative Perceptions of Patronizing Speech Addressed to Elders, Psychology and Aging, 6, pp. 442-450, 1991.

14. L. A. Wood and E. B. Ryan, Talk to Eiders: Social Structure, Attitudes, and Address,Ageing and Society, 11, pp. 167-188, 1991. 
15. M. M. Baltes and H. W. Wahl, The Behavior System of Dependency in the Elderly: Interaction With the Social Environment, in Aging, Health, and Behavior, M. G. Ory, R. P. Abeles, and P. D. Lipman (eds.), Sage, Newbury Park, California, 1992.

16. A. Lanceley, Use of Controlling Language in the Rehabilitation of the Elderly, Journal of Advanced Nursing, 10, pp. 125-135, 1985.

17. J. Rodin and E. J. Langer, Aging Labels: The Decline of Control and the Fall of Selfesteem, Journal of Social Issues, 36, pp. 12-29, 1980.

18. G. Kreps, Communication and Gerontology: Health Communication Training for Providers of Health Services to the Elderly, in Health Communication: Theory and Practice, G. Kreps and B. Thornton (eds.), Longman, New York, 1984.

19. World Health Organization, Health Promotion: A World Health Organization Document on the Concept and Principles, Canadian Public Health Association Digest, 8:6, pp. 101-102, 1984.

20. L. Green and J. Raebum, Health Promotion. What is It? What Will it Become?, Health Promotion, 3:2, pp. 151-159, 1988.

21. B. Compton and B. Galaway, Social Work Processes, The Dorsey Press, Illinois, 1989.

22. M. Lalonde, A New Perspective on Health of Canadians, Information Canada, Ottawa, 1974.

23. L. Pinder, From a New Perspective to the Framework: A Case Study on the Development of Health Promotion Policy in Canada, Health Promotion, 3:2, pp. 205-212, 1988.

24. I. Kickbusch, Health Promotion: A Global Perspective, Canadian Journal of Public Health, 77, pp. 321-326, 1986.

25. J. Epp, Achieving Health For All: A Framework for Health Promotion, Minister of Supply and Services, Ottawa, 1986.

26. M. R. Haug and M. G. Ory, Issues in Elderly Patient-Provider Interaction, Research on Aging, 9, pp. 3-44, 1987.

27. S. A. Fed orak and C. Griffin, Developing a Self-advocacy Program for S eniors: The Essential Component of Health Promotion, Canadian Journal on Aging, 5, pp. 269-277, 1986.

28. A. W. Franzke, The Effects of Assertiveness Training on Older Adults, The Gerontologist, 27, pp. 13-16, 1987.

29. A.E. Beisecker, Training Family Caregivers to Negotiate the Health Care System: The Context of Family Caregiver Training Materials, The Southwestern: The Journal of Aging for the Southwest, 7, pp. 29-45, 1991.

30. G. Kreps, Communication and Health Education, in Communication in Health Care Contexts: A Systems Perspective, E. B. Ray and L. Donohew (eds.), Lawrence Erlbaum Associates Inc., New Jersey, 1989.

31. R. Lubinski, The Environmental Role in Communication Skills and Opportunities of Older People, in Aging and Communication, C. Wilder and B. Weinstein (eds.), Haworth Press, New York, 1984.

32. A. E. Beisecker, Aging and the Desire for Information and Input in Medical Decisions: Patient Consumerism in Medical Encounters, The Gerontologist, 28:3, pp. 330-335, 1988.

33. E. J. Cassell, The Nature of Suffering and the Goals of Medicine, The New England Journal of Medicine, 306, pp. 639-645, 1982. 
34. E. Petch, Wise Use of Medications: A Health Promotion Approach to Community Programming for Safe Medication Use With and For Seniors, South Riverdale Community Health Centre, Toronto, 1992.

35. A. Norman, Triple Jeopardy: Growing Old in a Second Homeland, Centre for Policy on Ageing, London, 1985.

36. L. Driedger and N. L. Chappell, Aging and Ethnicity: Toward an Interface, Butt erworths, Toronto, 1987.

37. D. E. Gelfand and C. M. Barresi (eds.), Ethnic Dimensions of Aging, Springer Publications, New York, 1987.

38. L. K. Obler, S. de Santi, and J. Goldberger, Bilingual Dementia: Pragmatic Breakdown, in Dementia and Communication, R. Lubinski (ed.), Mosby, Philadelphia, 1991.

39. J. B. Wood, Communicating with Older Adults in Health Care Settings: Cultural and Ethnic Considerations, Educational Gerontology, 15, pp. 351-362, 1989.

40. M. J. MacLean and V. Sakadakis, Quality of Life in Terminal Care with Institutionalized Ethnic Elderly People, International Social Work, 32, pp. 209-221, 1989.

41. R. M. Coe and C. G. Prendergast, The Formation of Coalitions: Interaction Strat egies in Triads, Sociology of Health and Illness, 7, pp. 236-247, 1985.

42. L. Fratiglioni, M. Grut, Y. Forsell, M. Viitanen, M. Grafstrom, K. Holmen, K. Ericsson, L. Backman, A. Ahlbom, and B. Winblad, Prevalence of Alzheimer's Disease and Other Dementias in an Elderly Urban Population: Relationship with Age, Sex, and Education, Neurology, 41, pp. 1886-1892, 1991.

43. R. Lubinski (ed.), Dementia and Communication, Mosby, Philadelphia, 1991.

44. J. B. Orange, Perspectives of Family Members Regard ing Communication Changes, in Dementia and Communication, R. Lubinski (ed.), Mosby, Philadelphia, 1991.

45. L. Obler and M. Albert, Language and Aging, in Clinical Neurology of Aging, M. Albert (ed.), Oxford University Press, New Yore 1984.

46. G. Green, N. Linsk, and E. Pinkston, Modification of Verbal Behavior of the Mentally Impaired Elderly by Their Spouses, Journal of Applied Behavior and Analysis, 19, pp. 329-336, 1986.

47. M. Bourgeois, Communication Treatment for Adults with Dementia, Journal of Speech and Hearing Research, 34, pp. 831-844, 1991.

48. L Clark and K. Witte, Nature and Efficacy of Communication Treatment with Dementia, in Dementia and Communication, R. Lubinski (ed.), Mosby, Philadelphia, 1991.

49. D. W. Molloy and V. Mepham, Let Me Decide: The Health Care Directive That Speaks For You When You Can't, Penguin Books, Toronto, 1992.

Direct reprint requests to: (updated address):

Ellen B. Ryan, Ph.D.

McMaster Centre for Gerontological Studies, KTH-231

McMaster University

Hamilton, Ontario L8S 4M4

Canada 
Figure 1

\section{Communication Predicament Model}

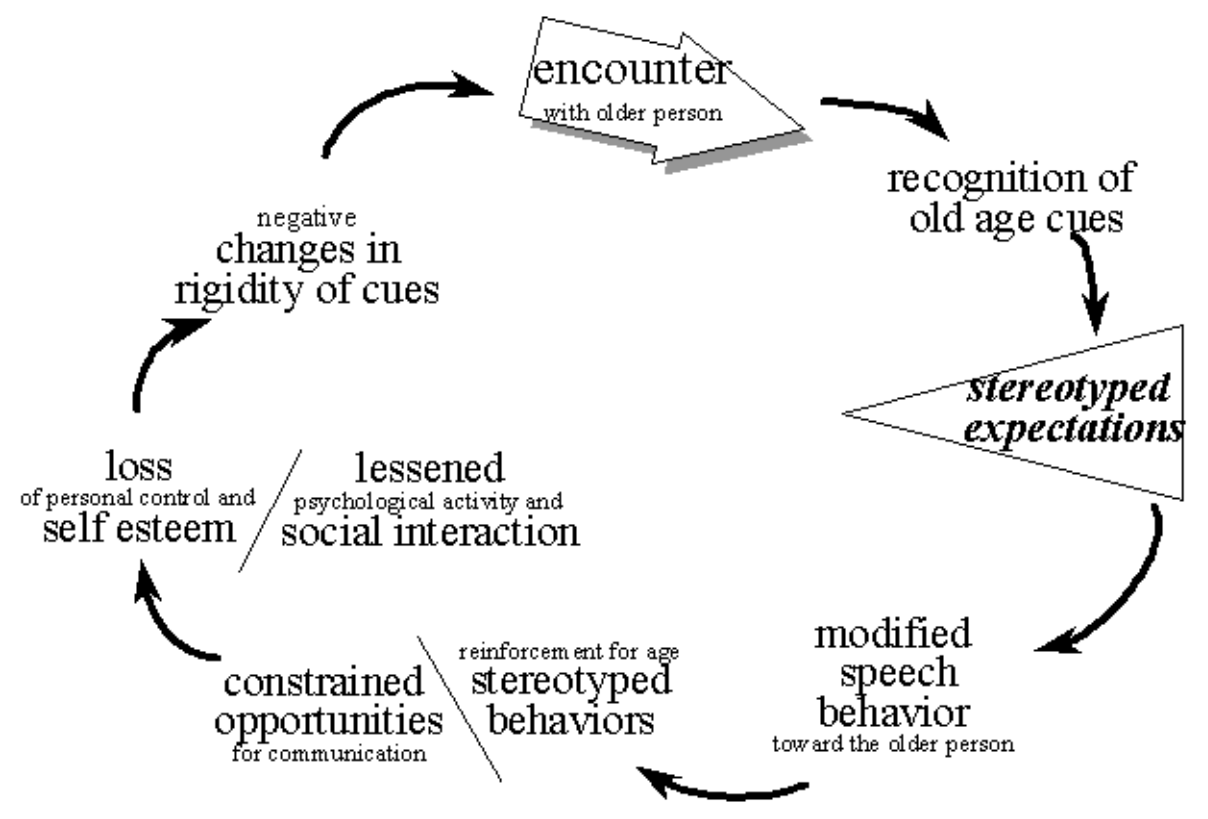

(Ryan, Giles, Bartolucci, \& Henwood, 1986)

Figure 2

\section{Communication Enhancement Model}

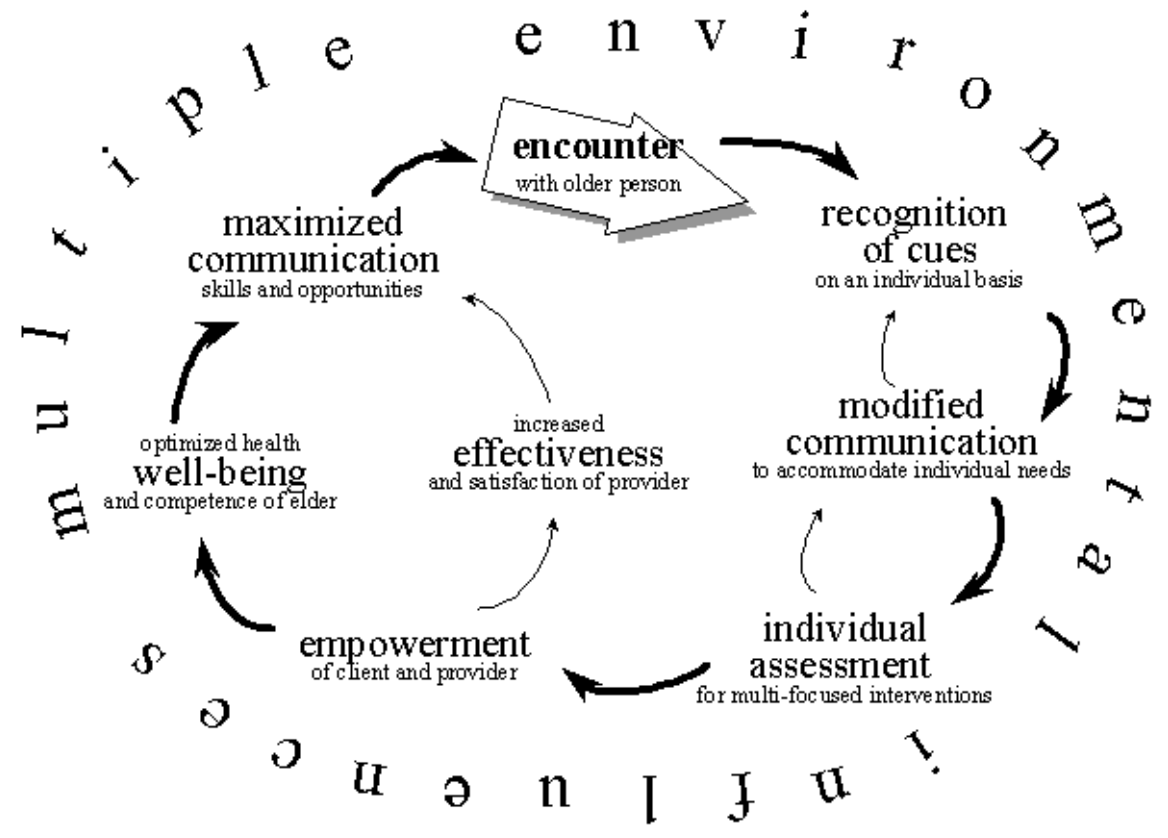

(Ryan, Meredith, MacLean, \& Orange, 1995) 